\title{
Effect of Lithium PFC Coatings on NSTX Density Control
}

H. W. Kugel, M. G. Bell, C. Bush, D. Gates, T. Gray, R. Kaita, B. Leblanc, R. Maingi, R. Majeski, D. Mansfield, D. Mueller, R. Raman, A. L. Roquemore, S. Sabbagh, C. H. Skinner, V. Soukhanovskii, T. Stevenson, L. Zakharov

\section{August 23, 2006}

Journal of Nuclear Materials 
This document was prepared as an account of work sponsored by an agency of the United States Government. Neither the United States Government nor the University of California nor any of their employees, makes any warranty, express or implied, or assumes any legal liability or responsibility for the accuracy, completeness, or usefulness of any information, apparatus, product, or process disclosed, or represents that its use would not infringe privately owned rights. Reference herein to any specific commercial product, process, or service by trade name, trademark, manufacturer, or otherwise, does not necessarily constitute or imply its endorsement, recommendation, or favoring by the United States Government or the University of California. The views and opinions of authors expressed herein do not necessarily state or reflect those of the United States Government or the University of California, and shall not be used for advertising or product endorsement purposes. 


\title{
Effect of Lithium PFC Coatings on NSTX Density Control
}

\author{
H. W. Kugel ${ }^{*}$, M.G. Bell ${ }^{a}$, C. Bush ${ }^{b}$, D. Gates ${ }^{\mathrm{a}}$, T. Gray ${ }^{a}$, R. Kaita ${ }^{a}$, B. Leblanc ${ }^{a}$,
}

R. Maingi ${ }^{b}$, R. Majeski ${ }^{a}$, D. Mansfield ${ }^{a}$, D. Mueller ${ }^{a}$, R. Raman $^{e}$, A. L. Roquemore ${ }^{a}$,

S. Sabbagh ${ }^{\text {c }}$, C. H. Skinner ${ }^{a}$, V. Soukhanovskii ${ }^{d}$, T. Stevenson ${ }^{a}$, L. Zakharov ${ }^{a}$

${ }^{a}$ Princeton Plasma Physics Laboratory, Princeton, NJ 08543, USA

${ }^{b}$ Oak Ridge National Laboratory, Oak Ridge, TN 37831, USA

${ }^{c}$ Columbia University, New York, NY 10027, USA

${ }^{d}$ Lawrence Livermore National Laboratory, Livermore, CA 94551, USA

${ }^{e}$ University of Washington, Seattle, WA 98195, USA

\begin{abstract}
Lithium coatings on the graphite plasma facing components (PFCs) in NSTX are being investigated as a tool for density profile control and reducing the recycling of hydrogen isotopes. Repeated lithium pellet injection into Center Stack Limited and Lower Single Null Ohmic Helium Discharges were used to coat graphite surfaces that had been pre-conditioned with Ohmic Helium Discharges of the same shape to reduce their contribution to hydrogen isotope recycling. The following deuterium NBI reference discharges exhibited a reduction in density by a factor of about 3 for limited and 2 for diverted plasmas respectively, and peaked density profiles. Recently, a lithium evaporator has been used to apply thin coatings on conditioned and unconditioned PFCs. Effects on the plasma density and the impurities were obtained by pre-conditioning the PFCs with ohmic helium discharges, and performing the first deuterium NBI discharge as soon as possible after applying the lithium coating.
\end{abstract}

PSI17 Subject Categories: Lithium; Particle control; Wall conditioning; Wall pumping; Impurity control.

JNM Keywords: C0600 Coatings; P0500 Plasma-materials interaction; S1300 Surface effects; I0100 Impurities.

PAC Numbers: 52.25.Vy; 52.40.-w; 52.40.Hf; 52.55.Fa.

* Corresponding author address: PPPL MS41, P. O. Box 451, Princeton NJ 08543.

* Corresponding author email: hkugel@pppl.gov.

* Presenting author: C. H. Skinner, email: cskinner@pppl.gov. 


\section{Introduction}

To achieve the NSTX goal of sustaining for several current-relaxation times its presently transient high-performance plasma regimes, we are investigating lithium plasmafacing components (PFCs) for particle and power handling. We have embarked on a phased, three-step approach to studying and developing the potential of lithium in NSTX: first by coating the present PFCs using lithium pellet injection, then using lithium evaporators, and finally, possibly, installing a liquid lithium divertor target. Taking the last step will depend on results to be obtained from the LTX experiment, formerly known as CDX-U, which recently succeeded in reducing the global recycling coefficient to less than $50 \%$ by using a toroidal tray of molten lithium as a limiter.[1] The techniques under development in NSTX and LTX may also be applicable to ITER and other future burning plasma fusion reactors.

Previously, achieving particle and impurity control in NSTX has involved a combination of high-temperature bakeout, boronization, and between-discharge helium glow discharge conditioning (HeGDC).[2] In light of NSTX needs for controlling the density rise and profile during long-duration H-modes, and in anticipation of similar ITER requirements, we have evaluated variations in the frequency and extent of boronization and the effectiveness of boronization with the PFCs hot $\left(250-300^{\circ} \mathrm{C}\right)$ and cold $\left(20^{\circ} \mathrm{C}\right)$ for facilitating access to H-modes. It was found that boronization on hot surfaces facilitated $\mathrm{H}$ mode access but slowly reverted to the behavior of boronization on cold surfaces, and that short boronizations, lasting $\sim 15 \mathrm{~min}$ and introducing about $2 \mathrm{~g}$ of deuterated trimethyl boron, followed by a comparable duration of HeGDC, applied in the morning before the start of daily experiments could restore and enhance good conditions, as evidenced by reduced impurity levels and ready access to the H-mode.[2] In the past year, we have also evaluated different techniques for helium plasma cleaning to reduce deuterium recycling, e.g. varying the duration of HeGDC between tokamak discharges, using fixed, wall-mounted vs. retractable, centrally located anodes for HeGDC, and testing the efficacy of using ohmic helium tokamak discharges between deuterium discharges. These comparisons were used to develop procedures which were successful in controlling impurities and the density rise during short-duration, up to about $1 \mathrm{~s}$, discharges. To achieve better density control in longerpulse discharges and more efficient current-drive for non-inductive current sustainment, more 
effective control of edge recycling is required, and this has motivated the lithium research that is now underway on NTSX.

The first step of the NSTX lithium program used lithium pellet injection (LPI) into standard NSTX discharges to produce lithium coatings on the plasma contact areas of the PFCs. This technique was pioneered on TFTR where reduced recycling and significantly enhanced fusion performance were obtained by starting with a limiter thoroughly depleted of hydrogen isotopes and applying lithium with three deposition methods: lithium pellet injection, evaporation of lithium from an oven, and lithium micro-droplet introduction (DOLLOP) directly into low density plasmas.[3] During and since the TFTR experiments, the LPI technique was investigated in other devices with divertors, notably C-MOD, DIII-D and TdeV. However, without the thorough wall degassing applied in TFTR, none of these experiments yielded similar performance improvement other than a small decrease in impurities.[4] The goal of these NSTX LPI experiments was to build on the TFTR lithium experience, to investigate the effect of the wall conditions on the success of LPI and to reproduce the recycling reduction and improved density control. These LPI investigations in NSTX have been followed by recent experiments using a lithium evaporator to deposit lithium films on the lower divertor and the center stack regions of the PFCs.

\section{Lithium pellet injection into discharges not preceded by helium discharge conditioning}

In the initial NSTX LPI experiment, lithium pellets were injected during the plasma current ramp-up in ohmic discharges and, in NBI discharges, both preceding and during the NBI heating pulse. Pellets were also injected late into discharges preceding NBI discharges. In this experiment, a total of $34 \mathrm{mg}$ of lithium was injected using $2 \mathrm{mg}$ pellets into 16 discharges in Lower Single Null (LSN) and Double-Null (DN) divertor configurations. Each of these discharges was preceded by $7-11 \mathrm{~min}$ of HeGDC to remove adsorbed fuel gas from PFC surfaces but no helium conditioning discharges were used. During the LPI sequence, the C III luminosity was approximately constant, but the O V luminosity fell steadily to about $50 \%$ of its original level. Long after these LPI experiments, residual Li I luminosity was 
observed from the center stack region. During NBI heating of the LSN discharges, the pellets ablated near the plasma edge and much lithium appeared to be transported not only to the lower divertor, but also to the upper divertor along open field lines, as indicated by a TV image of the Li I emission. During NBI in DN discharges, the pellets also ablated near the edge but the lithium exhibited transport more uniformly to both divertors along open field lines. In both LSN and DN plasmas, the rise in the line-average density during NBI was not obviously changed by the lithium deposition. When pellets were injected into the ohmic phase of discharges pre-heated with NBI, the pellet penetration depth was sensitive to a $10 \mathrm{~ms}$ change in NBI turn-off time relative to pellet arrival. After LPI, some discharges continued for their programmed duration, but others collapsed slowly; locked modes seemed more prevalent as the lithium deposition increased.

\section{Lithium pellet injection experiments in Center Stack Limited discharges preceded by helium discharge conditioning}

In the next NSTX lithium experiments, Center-Stack Limited (CSL), ohmic helium conditioning discharges (0.5 MA, 0.45 T) were used to degas the PFCs on the center stack. Each of these discharges was preceded by $7-11$ mins of HeGDC to augment the degassing process. Fig. 1 shows how the $\mathrm{D}_{\alpha}$ emission from the center stack region decreased with discharge number until after about 8 discharges a baseline level was reached beyond which only a relatively small change per discharge occurred. This behavior, which has been observed in repeated, widely spaced, experiments, may be understood as due to depletion of the primary contact region combined with a continuing residual outgassing from the outer graphite passive plate surfaces, RF antenna cavities, and the main chamber outer wall within range of recycling from fast change-exchange neutrals. After the first 8 helium discharges shown in Fig. 1, 3 deuterium, CSL, NBI-heated reference discharges (0.9 MA, 0.45 T) were run to evaluate the wall conditions. The $\mathrm{D}_{\alpha}$ signal exhibited a monotonic increase in this sequence as the conditioned surfaces were "re-gassed" by these discharges. Four ohmic helium conditioning discharges were then applied to degass the PFC surfaces once more. Lithium pellets, with masses $1.7,3.4$, or $5.0 \mathrm{mg}$, were then injected into 9 of the next 12 similar ohmic, helium discharges. During this sequence of discharges with LPI, the $\mathrm{D}_{\alpha}$ luminosity (Fig. 1) continued to decrease slightly ( 25\%). Fig. 2 shows how the Li I 
luminosity from the CS region increased as the lithium injection accumulated to a total of $30 \mathrm{mg}$. After this lithium coating sequence, three reference NBI-heated CSL discharge (Fig. 3) were performed. Following the initial gas puff, the first of these discharges exhibited a reduction in the volume-average density of about a $33 \%$ compared to the reference shot prior to the lithium coating. After reaching the minimum in density, the rate of density rise in this first discharge was approximately equal to the rate of fuelling from the NBI. The second reference discharge reverted to the density level before lithium application, and by the third discharge there was sufficient recycling from the walls to exceed the pre-lithium density. After this sequence, another $24 \mathrm{mg}$ of lithium was deposited using the same ohmic helium discharges, and almost identical density behavior was observed in the subsequent CSL NBI reference discharges. These results are similar to the experience in TFTR in which the benefit of lithium coating by LPI was observed only if the discharges were preceded by adequate degassing of the graphite PFC surfaces using helium discharges.

\section{Lithium pellet injection experiments in Lower Single Null discharges preceded by helium discharge conditioning}

In a subsequent NSTX experiment, LSN divertor, ohmic helium discharges were used to condition the lower divertor target. This was followed by a reference LSN, deuterium plasmas (0.9 MA, 0.45 T) with NBI. LPI into a sequence of similar helium discharges was then used to introduce a total of about $25 \mathrm{mg}$ of $\mathrm{Li}$. As this deposition progressed, the $\mathrm{Li}$ I line emission from the lower divertor region increased. Finally, a series of three reference plasmas with NBI was performed (Fig. 4). In the first of these, the density was reduced about $50 \%$ from the reference discharge prior to the lithium coating. The reduction in density was less on the second and absent on the third shot, again consistent with exhausting the capacity of the deposited lithium to absorb deuterium. In this experiment the rise in density increased with the introduction of NBI core fueling but in a more complex manner than as in the CSL experiments described above. 


\section{Initial experiments on evaporated lithium surfaces}

A lithium evaporation oven was mounted on an upper divertor port and aimed downward at an angle of $22^{\circ}$ from the vertical to provide coverage over the lower divertor and center stack regions. Five evaporations were performed in amounts ranging from 14 to $640 \mathrm{mg}$, for evaporation times ranging from 68 to 387 mins. The amount of lithium evaporated from the oven was calculated from the temperature vs. time of the oven. The graphite PFC's were at room temperature throughout the evaporation. The time between end of evaporation and the next discharge ranged from 23 to 140 mins. During this delay, some fraction of the deposited lithium may have reacted with the vacuum residual gas components or have intercalated into the graphite PFC surface. [5-7] These 5 evaporations were preceded by different wall conditioning procedures, either none, HeGDC, or ohmic helium discharges.

The initial relatively small evaporations, applied slowly over long times on essentially unconditioned surfaces, and with relatively long times between end of evaporation and next discharge produced no change in the density evolution of a reference discharge. However, effects were obtained by running $6-8$ ohmic helium discharges first, evaporating a relatively large amount of lithium ( $400 \mathrm{mg}$ ) onto the conditioned surfaces as fast as possible (68 min), and then performing the first reference discharge as soon as possible thereafter (23 mins). The first of a sequence of 4 NBI reference discharges following this evaporation exhibited approximately a $29 \%$ decrease in density relative to the comparison discharge, peaked density profiles, electron temperatures of $2 \mathrm{keV}$ for $1 \mathrm{MW} \mathrm{NBI}$, and an energy confinement time of $\sim 90 \mathrm{~ms}$. Fig. 5 shows profiles from this and the next discharge in the sequence. Through this sequence the emission from hydrogen-like oxygen time-integrated through each discharge was being measured by an extreme ultra-violet spectrometer. The O VIII level was essentially the same for the first post-lithium discharge (120474) as before the evaporation. However, over the following 3 NBI shots the signal fell to the noise background indicating a significant reduction in near-core oxygen impurities.

An experiment was also conducted to assess whether the apparent effects of the lithium coating on the density could be attributable solely to the helium pre-conditioning of the PFCs. Fig. 6 compares the profiles of discharges before and after a sequence of helium conditioning 
shots, showing that these discharges alone were responsible for an approximately $18 \%$ decrease in density relative to the comparison discharge. Thus, under the present experimental conditions, helium conditioning appears to produce a similar pumping rate for the graphite surfaces to lithium deposited on the conditioned graphite surfaces.

\section{Discussion}

In Lithium pellet injection experiments into discharges not preceded by helium discharge conditioning, the total injected mass of $25-34 \mathrm{mg}\left(2.2-3.0 \times 10^{21} \mathrm{Li}\right.$ atoms $)$ would have an average thickness of $\sim 1.5 \mathrm{~nm}$ if spread uniformly over the area of the PFCs in NSTX, or $\sim 30 \mathrm{~nm}$ if deposited uniformly over the center stack, or $\sim 150 \mathrm{~nm}$ if deposited in a $10 \mathrm{~cm}$ wide annulus at the outer strike point on the lower divertor. The densities of the reference discharges were not changed by lithium deposition of this amount on surfaces which had not been depleted of fuel gas by helium conditioning discharges. However, in both the CSL and LSN experiments involving lithium deposition after helium discharge conditioning, a significant reduction in density was observed in the first reference discharge, a small reduction on the second and essentially no effect on the third shot. This is consistent with reaction of the deposited lithium with the deuterium gas puffed into the plasma, typically about $1.0 \times 10^{21} \mathrm{D}$ per shot, to form LiD. Our initial experiments applying D discharges to evaporated lithium surfaces demonstrate the effectiveness of the thin lithium coating pumping on deuterium-depleted graphite surfaces and the need for prompt application of the reference discharge. The lithium coatings used in these experiments appear sensitive not only to interactions with residual fuel gases in the graphite substrate but also to time after the deposition since interactions with vacuum vessel residual gas components $\left(\mathrm{H}_{2}\right.$, $\mathrm{D}_{2} \mathrm{H}_{2} \mathrm{O}, \mathrm{D}_{2} \mathrm{O}, \mathrm{N}_{2}, \mathrm{CO}$, and $\mathrm{CO}_{2}$ ) and intercalation of the lithium into the graphite [5-7] can occur. These results are consistent with TFTR results [3] and laboratory experiments [5-7]. This sensitivity to substrate conditions may change as a lithium coating thickness is increased further. The effect on density control of increasing the lithium deposition amount, the rate of deposition, the duration before the reference discharge, and the discharge start-up conditions are under investigation. The installation of a liquid divertor in NSTX will depend on results to be obtained from the LTX experiment, formerly known as CDX-U, where a substantial 
reduction in recycling and a complete suppression of oxygen contamination of the plasma was produced by using a toroidal tray of molten lithium as a limiter.[1]

\section{Acknowledgment}

This work is supported by U.S. Department of Energy Contracts, DE-AC0276CH03073, DE-AC05-00OR22725, DE-FG02-99ER54523 and DE-FG03-99ER54361, W7405-ENG-48 (with UC, LLNL). We wish to acknowledge the technical contributions of the NSTX Engineering and Research Teams. 


\section{References}

[1] R. Majeski et al., Nucl. Fusion, 45 (2005) 519.

[2] H.W. Kugel, et al., J. Nucl. Mater., 337-339 (2005) 495.

[3] D.K. Mansfield, et al., Nucl. Fus., 41 (2001) 1823.

[4] J.T. Hogan, C.E.Bush, C.H.Skinner, Nucl. Fusion, 37 (1997) 705, and references therein.

[5] H. Sugai, et al., J. Nucl. Mater., 220-222 (1995) 254.

[6] N. Itou, et al., J. Nucl. Mater., 290-293 (2001) 281.

[7] H. Yagi, et al., J. Nucl. Mater., 313-316 92003) 284. 


\section{Figure Captions}

Fig. 1. Evolution of the representative level of $D_{\alpha}$ luminosity from a sequence of 8 ohmic conditioning discharges ( $0.5 \mathrm{MA}, 0.45 \mathrm{~T}$ ), followed by 3 deuterium reference discharges, 4 additional ohmic clean-up discharges, and then 12 ohmic helium discharges, 9 of which with LPI of either $1.7,3.4$, or $5.0 \mathrm{mg}$. A small decrease in $\mathrm{D}_{\alpha}$ luminosity was evident in the discharges with LPI prior to the arrival of the LPI.

Fig. 2. The Li I luminosity from the center stack increased as the total lithium injection increased to $30 \mathrm{mg}$.

Fig. 3. Initial CSL NBI deuterium reference discharge (0.9 MA, $0.45 \mathrm{~T})$ following $30 \mathrm{mg}$ of lithium deposition exhibited a decrease in density of about $33 \%$ after termination of the initial gas puff. On the second discharge, the effect was less while on the third, the density was higher than before. Approximately $3.5 \mathrm{mg}$ of $\mathrm{D}_{2}$ gas fueling was injected into each discharge.

Fig. 4. Initial LSN NBI deuterium reference discharge (0.8 MA, 0.45 T) following $25 \mathrm{mg}$ of lithium deposition on lower divertor exhibited a decrease in density by about $50 \%$. Approximately $3.5 \mathrm{mg}$ of $\mathrm{D}_{2}$ gas fueling is injected into each discharge.

Fig. 5. Comparison of Thomson scattering profiles for NBI D reference discharges before (shot 120464) and after $(120474,5)$ lithium deposition following ohmic helium conditioning discharges. Pumping by the lithium coating on the conditioned graphite PFCs exhibited almost a $29 \%$ decrease in density relative to the comparison discharge

Fig. 6. Comparison of Thomson scattering profiles for NBI D reference discharges before (120496) and after $(120505,8)$ ohmic helium conditioning discharges applied to the graphite plasma facing surfaces. Pumping by the degassed graphite exhibited about an $18 \%$ decrease in density relative to the comparison discharge. 


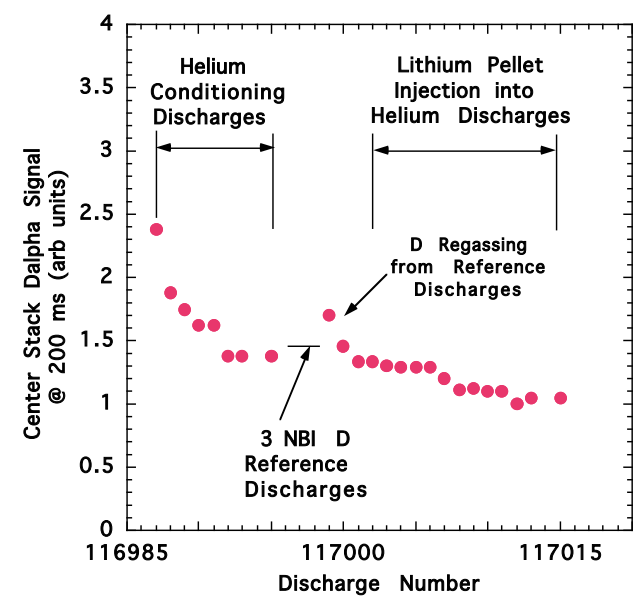

Fig. 1. Evolution of the representative level of $D_{\alpha}$ luminosity from a sequence of 8 ohmic conditioning discharges ( $0.5 \mathrm{MA}, 0.45 \mathrm{~T})$, followed by 3 deuterium reference discharges, 4 additional ohmic clean-up discharges, and then 12 ohmic helium discharges, 9 of which with LPI of either $1.7,3.4$, or $5.0 \mathrm{mg}$. A small decrease in $\mathrm{D}_{\alpha}$ luminosity was evident in the discharges with LPI prior to the arrival of the LPI. 


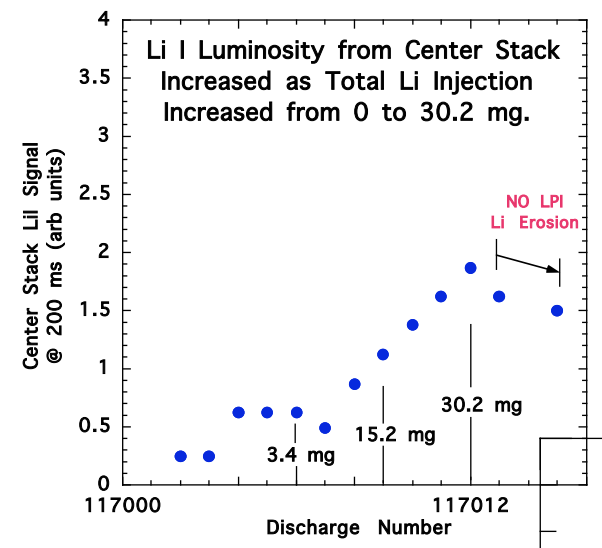

Fig. 2. The Li I luminosity from the center stack increased as the total lithium injection increased to $30 \mathrm{mg}$. 


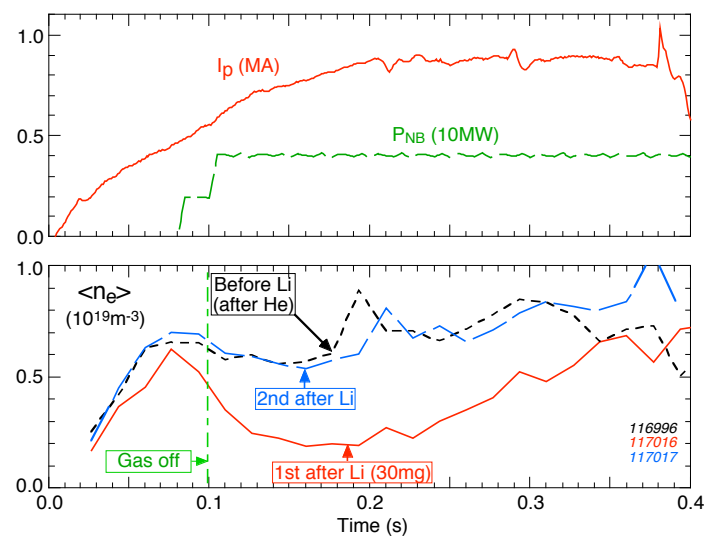

Fig. 3. Initial CSL NBI deuterium reference discharge (0.9 MA, $0.45 \mathrm{~T})$ following $30 \mathrm{mg}$ of lithium deposition exhibited a decrease in density of about $33 \%$ after termination of the initial gas puff. On the second discharge, the effect was less while on the third, the density was higher than before. Approximately $3.5 \mathrm{mg}$ of $\mathrm{D}_{2}$ gas fueling was injected into each discharge. 
P1-79

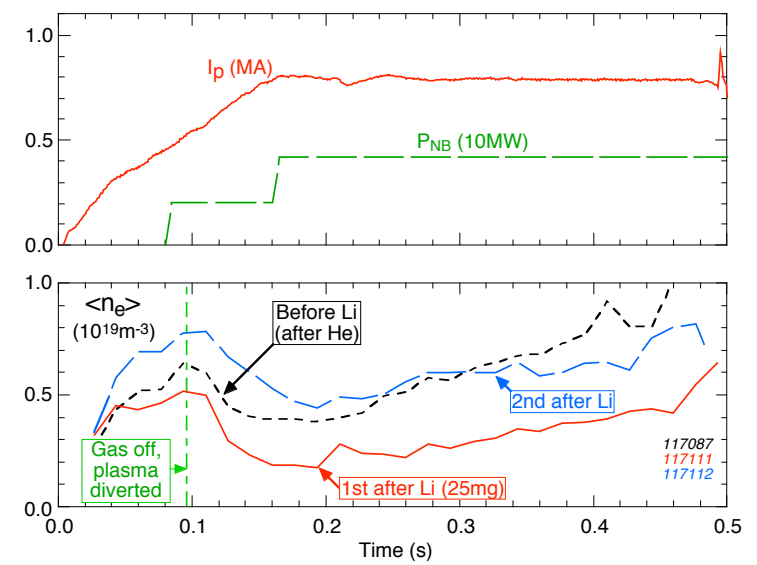

Fig. 4. Initial LSN NBI deuterium reference discharge (0.8 MA, 0.45 T) following $25 \mathrm{mg}$ of lithium deposition on lower divertor exhibited a decrease in density by about $50 \%$. Approximately $3.5 \mathrm{mg}$ of $\mathrm{D}_{2}$ gas fueling is injected into each discharge. 
P1-79
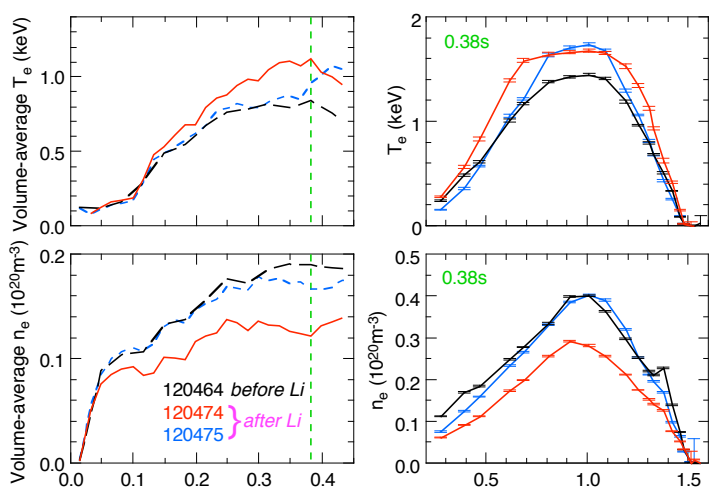

Fig. 5. Comparison of Thomson scattering profiles for NBI D reference discharges before (shot 120464) and after $(120474,5)$ lithium deposition following ohmic helium conditioning discharges. Pumping by the lithium coating on the conditioned graphite PFCs exhibited almost a $29 \%$ decrease in density relative to the comparison discharge 
P1-79
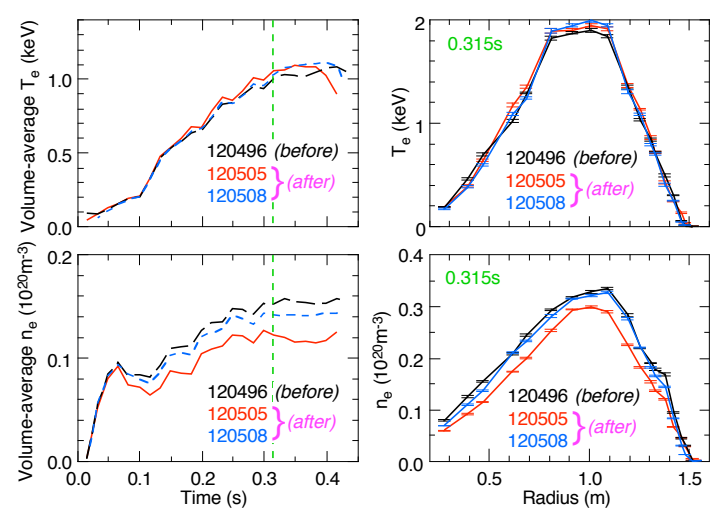

Fig. 6. Comparison of Thomson scattering profiles for NBI D reference discharges before (120496) and after $(120505,8)$ ohmic helium conditioning discharges applied to the graphite plasma facing surfaces. Pumping by the degassed graphite exhibited about an $18 \%$ decrease in density relative to the comparison discharge. 